\section{TATRA \\ MOUNTaiNS \\ Mathematical Publications}

DOI: $10.2478 / \mathrm{tmmp}-2014-0034$

Tatra Mt. Math. Publ. 61 (2014), 163-173

\title{
THREE-POLAR SPACE OVER THE SEMI-FIELD OF DOUBLE NUMBERS
}

\author{
TOMÁš GREGOR
}

\begin{abstract}
Multi-polar space is a generalization of the notion of vector space. In this paper, we deal with a three-polar vector space over a semi-field of double (hyperbolic complex) numbers. We introduce and study operations of addition and multiplication such that they form a commutative ring with unit on the three-polar space.
\end{abstract}

\section{Introduction}

As far as we know, the idea of multi-polarity is relatively new and comes from physics, cf. 6]. There are, e.g., $K$-polar oscillators, magnets, $K \in \mathbb{N}$. The principle of multi-polarity can be easily observed in the case of $K$-phase electric current, $K \in \mathbb{N}$. Particularly, if $K=3$, we say about three-phase current. The sum of all three phases of equal amplitude in three-phase current is zero at each moment. We call this fact to be the cancellation law in this paper.

Another source of motivation for multi-polarity study is a color vision. If we deal with a classical RGB model of colors, then polarities are represented by red, green and blue. The mixture of these basic colors with the same intensities makes a gray color (from white to black, depending on intensity). So, the cancellation law can be understood as "gray color" in this model.

Multi-polarity can be imagined as a "colouring" of the black-and-white mathematics. A mathematical description of multi-polarity in the plane is introduced in [4. There is presented the complex plane as a three-polar space.

Every Euclidean space (with two standard polarities "+" and "-") is a multipolar space, where the real field is replaced by a semi-field $[0, \infty)$. Remind the semi-field definition.

(C) 2014 Mathematical Institute, Slovak Academy of Sciences.

2010 Mathematics Subject Classification: 13F15, 16S99, 12K10, 30 G35.

Keywords: multi-polar space, semi-field, double numbers.

This paper was supported by the grant VEGA 2/0178/14 and by the project of SAS and NASU "Vector valued measures and integration in polarized vector spaces". 


\section{TOMÁŠ GREGOR}

Definition 1.1. Let $X$ be a set with two binary operations + and $\cdot$. The system $(X,+, \cdot)$ is said to be a semi-field if $(X,+)$ is a commutative semi-group, $(X, \cdot)$ is a commutative multiplicative group, and multiplication distributes over addition.

Elements of a semi-field $X$ are called non-polar or neutral elements.

Let $\mathbb{A}=\{A, B, C\}$ be a set of three mappings from the given semi-field $X$ to a set $Y$. The mappings are called polar operators, or, poles. According to the number of polar operators we say about three-polar space.

In this paper we consider a binary operation $*$ on $\mathbb{A}$ given by the Latin square

\begin{tabular}{c|ccc}
$*$ & $A$ & $B$ & $C$ \\
\hline$A$ & $A$ & $B$ & $C$ \\
$B$ & $B$ & $C$ & $A$ \\
$C$ & $C$ & $A$ & $B$
\end{tabular}.

There is a neutral element $A$ with respect to the operation $*$. Next, we assume that $A(X), B(X), C(X)$ are isomorphic copies of $X$ and

$$
A(0)=B(0)=C(0),
$$

where 0 is a neutral element in the semi-group $(X,+)$. The images $A(X), B(X)$ and $C(X)$ are called polar axes. The condition (2) shows that all polar axes intersect in a single point which can be viewed as an "origin" of the multi-polar space.

In the paper [4, the semi-field of all non-negative real numbers with usual addition and multiplication was used to construct the multi-polar spaces. The resulting multi-polar spaces with arithmetic operations of addition, subtraction, multiplication and division are all isomorphic to the system of complex numbers. In this paper we bring a three-polar space over a more general semi-field, a subset of the so-called double numbers.

We construct new quaternion-like systems. An interesting question is about a generalization to octonions. Octonions are used in physics, cf. [2], [3, [7].

\section{A semi-field of double numbers}

In this paper, we deal with the system of double numbers 11 We will denote the system of double numbers as $\mathbb{D}$.

\footnotetext{
${ }^{1}$ There is no standard terminology for "double" numbers; we can find the following terms in literature: hyperbolic complex numbers, hyperbolic numbers, semi-complex numbers, splitcomplex numbers, perplex numbers.
} 


\section{THREE-POLAR SPACE OVER THE SEMI-FIELD OF DOUBLE NUMBERS}

Double numbers are similar to classical complex numbers. Arithmetic operations of double numbers $\left(a_{1}, a_{2}\right),\left(b_{1}, b_{2}\right) \in \mathbb{R}^{2}$ are as follows

$$
\begin{aligned}
\left(a_{1}, a_{2}\right)+\left(b_{1}, b_{2}\right) & =\left(a_{1}+b_{1}, a_{2}+b_{2}\right), \\
\left(a_{1}, a_{2}\right)-\left(b_{1}, b_{2}\right) & =\left(a_{1}-b_{1}, a_{2}-b_{2}\right), \\
\left(a_{1}, a_{2}\right) \cdot\left(b_{1}, b_{2}\right) & =\left(a_{1} b_{1}+a_{2} b_{2}, a_{1} b_{2}+a_{2} b_{1}\right),
\end{aligned}
$$

and if $b_{1}^{2} \neq b_{2}^{2}$, then

$$
\frac{\left(a_{1}, a_{2}\right)}{\left(b_{1}, b_{2}\right)}=\left(\frac{a_{1} b_{1}-a_{2} b_{2}}{b_{1}^{2}-b_{2}^{2}}, \frac{a_{2} b_{1}-a_{1} b_{2}}{b_{1}^{2}-b_{2}^{2}}\right) .
$$

All operations on the right sides are usual operations of real numbers. We will denote operations on $\mathbb{R}$ and $\mathbb{R}^{2}$ alike.

It is easy to check that double numbers form a commutative ring with unit but not a semi-field. If we take the set $\left\{\left(x_{1}, x_{2}\right) \in \mathbb{R}^{2} \mid x_{1} \geq 0, x_{2}=0\right\}$, then double numbers are reduced on the semi-field of non-negative real numbers. There exists also a non-trivial subset of double numbers which is closed under addition, multiplication and division. It is a set $\mathbb{D}_{+}^{0}=\left\{\left(x_{1}, x_{2}\right) \in \mathbb{R}^{2} \mid x_{1}>\right.$ $\left|x_{2}\right|$ or $\left.x_{1}=x_{2}=0\right\}$. Obviously, the system $\left(\mathbb{D}_{+}^{0},+, \cdot\right)$ is a semi-field. For more information about double numbers, cf., e.g., [1], [5].

We introduce the following definition for the lemma of "existence of the square of the difference".

Definition 2.1. Let $a=\left(a_{1}, a_{2}\right) \in \mathbb{D}_{+}^{0}$ and $b=\left(b_{1}, b_{2}\right) \in \mathbb{D}_{+}^{0}$. We will say that $a, b$ are in the relation $\stackrel{+}{\sim}(\bar{\sim}), a \stackrel{ \pm}{\sim} b(a \bar{\sim} b)$, if $a_{1}+a_{2}=b_{1}+b_{2}\left(a_{1}-a_{2}=b_{1}-b_{2}\right)$.

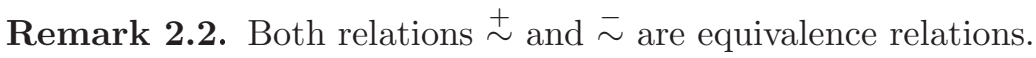

Lemma 2.3. Let $a=\left(a_{1}, a_{2}\right) \in \mathbb{D}_{+}^{0}$ and $b=\left(b_{1}, b_{2}\right) \in \mathbb{D}_{+}^{0}$. Then

1. $(a-b)^{2} \in \mathbb{D}_{+}^{*}=\mathbb{D}_{+}^{0} \cup\left\{\left(x_{1}, x_{2}\right) \in \mathbb{R}^{2}\left|x_{1}=\right| x_{2} \mid\right\}$,

2. $(a-b)^{2} \in \mathbb{D}_{+}=\mathbb{D}_{+}^{0} \backslash\{(0,0)\}$ if and only if $a \psi^{\ddagger} b$ and $a \not z b$.

Proof. There holds for $a, b \in \mathbb{D}$

$$
(a-b)^{2}=\left(\left(a_{1}-b_{1}\right)^{2}+\left(a_{2}-b_{2}\right)^{2}, 2\left(a_{1}-b_{1}\right)\left(a_{2}-b_{2}\right)\right) .
$$

1. Obviously, $\left|a_{1}-b_{1}\right|^{2}+\left|a_{2}-b_{2}\right|^{2} \geq 2\left|a_{1}-b_{1}\right|\left|a_{2}-b_{2}\right|$ for all $a_{1}, a_{2}, b_{1}, b_{2} \in \mathbb{R}$ which implies that $(a-b)^{2} \in \mathbb{D}_{+}^{*}$.

2. $(a-b)^{2} \in \mathbb{D}_{+} \Longleftrightarrow\left(a_{1}-b_{1}\right)^{2}+\left(a_{2}-b_{2}\right)^{2}>2\left|\left(a_{1}-b_{1}\right)\left(a_{2}-b_{2}\right)\right|$

$$
\begin{aligned}
& \Longleftrightarrow\left(\left|a_{1}-b_{1}\right|-\left|a_{2}-b_{2}\right|\right)^{2}>0 \\
& \Longleftrightarrow\left|a_{1}-b_{1}\right| \neq\left|a_{2}-b_{2}\right|
\end{aligned}
$$

$\Longleftrightarrow a_{1}+a_{2} \neq b_{1}+b_{2}$ and $a_{1}-a_{2} \neq b_{1}-b_{2} \Longleftrightarrow a \neq b$ and $a \Varangle b$. 


\section{Three-polar space over double numbers}

The next step in construction of a three-polar space is to find a suitable system of three polar operators. Let us consider three poles $A, B, C$ from the semi-field $\mathbb{D}_{+}^{0}$ to a set $Y$ and an operation $*$ given by the Latin square (11). For convenience, denote

$$
X:=\mathbb{D}_{+}^{0}, \quad X_{+}:=X \backslash\{(0,0)\}=\mathbb{D}_{+} .
$$

Now let us consider the element $(a, b, c) \in X^{3}$. It can be very formally written as a sum

$$
(a, b, c)=A a+B b+C c .
$$

\subsection{Cancellation law, "null of addition"}

Definition 3.1. Let $(u, v, w),(x, y, z) \in X^{3}$. We will say that the triple $(u, v, w)$ is equal to the triple $(x, y, z)$ in the sense of cancellation law ("null of addition"), we write $(u, v, w) \cong(x, y, z)$, if there exists $d \in X$, such that $(u+d, v+d, w+d)=$ $(x, y, z)$ or $(u, v, w)=(x+d, y+d, z+d)$.

\section{Remark 3.2.}

1. Relation $\cong$ is an equivalence relation.

2. If $(a, b, c) \in X^{3}$, then $(a, b, c) \cong(0,0,0) \Longleftrightarrow a=b=c$. Formally, for all $a \in X$

$$
A a+B a+C a \cong(0,0,0) .
$$

3. If $a, b, c \in \mathbb{R}^{2}$, there exists $d \in X$ such that $(a+d, b+d, c+d) \in X^{3}$, for example, $d=\left(d_{1}, 0\right)$, where $d_{1}=1+\max \left\{\left|x_{1}\right|+\left|x_{2}\right|,\left|y_{1}\right|+\left|y_{2}\right|,\left|z_{1}\right|+\left|z_{2}\right|\right\}$.

\subsection{Operations in the three-polar space}

We introduce operations analogical to the classical operations of addition, subtraction, conjugation, multiplication and division. All these operations are defined on $X^{3}$.

Addition. For $(u, v, w),(x, y, z) \in X^{3}$,

$$
(u, v, w) \oplus(x, y, z):=(u+x, v+y, w+z) .
$$

Clearly, the operation $\oplus$ is associative and commutative.

Subtraction. For all $x, y, z \in X$ and due to the cancellation law, formally

$$
\begin{aligned}
& -A x=B x+C x, \\
& -B y=A y+C y, \\
& -C z=A z+B z .
\end{aligned}
$$

We define the operation of subtraction as follows

$$
\begin{aligned}
(u, v, w) \ominus(x, y, z) & =(u, v, w) \oplus(y+z, x+z, x+y) \\
& =(u+y+z, v+x+z, w+x+y) .
\end{aligned}
$$




\section{THREE-POLAR SPACE OVER THE SEMI-FIELD OF DOUBLE NUMBERS}

It is obvious that $\ominus(x, y, z)$ is just an inverse element to $(x, y, z)$ with respect to $\oplus$ because

$$
(x, y, z) \ominus(x, y, z)=(x+y+z, x+y+z, x+y+z) \cong(0,0,0) .
$$

Multiplication. We define the operation of multiplication polynomial-like ("each one with each one") using the table for operation *, i.e.,

$$
\begin{aligned}
(u, v, w) \odot(x, y, z) \\
=(A u+B v+C w) \odot(A x+B y+C z) \\
=(A * A)(u x)+(A * B)(u y)+(A * C)(u z)+(B * A)(v x)+(B * B)(v y) \\
\quad+(B * C)(v z)+(C * A)(w x)+(C * B)(w y)+(C * C)(w z) \\
=A(u x+v z+w y)+B(u y+v x+w z)+C(u z+v y+w x) \\
=(u x+v z+w y, u y+v x+w z, u z+v y+w x) .
\end{aligned}
$$

This operation is associative and commutative and its neutral element is $((1,0),(0,0),(0,0))$. It is also easy to see that the operation of multiplication distributes over addition.

The special case of this operation is multiplication by scalar,

$$
k(u, v, w)=(k, 0,0) \odot(u, v, w)=(k u, k v, k w), \quad \text { where } \quad k \in X .
$$

Conjugation. This operation is expressed in terms of three-polar space in the following way

$$
(u, v, w)^{*}=(u, w, v) .
$$

It can be easily to check that operation of conjugation has the following properties.

Lemma 3.3. Let $(u, v, w) \in X^{3},(x, y, z) \in X^{3}$. Then there holds:

1. $\left((u, v, w)^{*}\right)^{*}=(u, v, w)$,

2. $((u, v, w) \oplus(x, y, z))^{*}=(u, v, w)^{*} \oplus(x, y, z)^{*}$,

3. $((u, v, w) \odot(x, y, z))^{*}=(u, v, w)^{*} \odot(x, y, z)^{*}$,

4. $(u, v, w) \odot(u, v, w)^{*} \cong(d, 0,0)$ for some $d \in X_{+}$if and only if it is not true that $u \stackrel{ \pm}{\sim} \stackrel{ \pm}{\sim} w$ or $u \sim v \sim w$.

Proof.

1.-3. are trivial.

4. Let $(u, v, w) \in X^{3}$. We have

$$
(u, v, w) \odot(u, v, w)^{*}=(u u+v v+w w, u w+v u+w v, u v+v w+w u),
$$

thus it is sufficient to prove that

$$
d=\left(u^{2}+v^{2}+w^{2}\right)-(u w+v u+w v) \in X_{+}
$$

if and only if it is false that $u \stackrel{+}{\sim} v \stackrel{+}{\sim} w$ or $u \succsim v \bar{\sim} w$. 


\section{TOMÁŠ GREGOR}

There holds that

$$
d=\frac{1}{2}\left[(u-v)^{2}+(v-w)^{2}+(w-u)^{2}\right] .
$$

If $d \in X_{+}$, then at least one of elements $(u-v)^{2},(v-w)^{2},(w-u)^{2}$ belongs to $X_{+}$, for example, let there be $(u-v)^{2}$, and thus $u \neq v$ and $u \not v$, by Lemma 2.3 .

Conversely, let the statement $u \stackrel{+}{\sim} v \stackrel{+}{\sim} w$ or $u \succsim v \succsim w$ not be true. Then there exists a pair

$$
a, b \in\{u, v, w\} \text { such that } a \not b \text { and } a \not b \text {. }
$$

Let $u \not w$, without loss of generality. If $u \not z$, then $a=u, b=w$; if $v \neq w$ and $v \not w$, then $a=v, b=w$. Otherwise $u \not v$ and $u \Varangle v$, thus $a=u, b=v$. By Lemma 2.3, $(a-b)^{2}$ belongs to $X_{+}$and also $d \in X_{+}$.

Division. Due to the previous lemma, and the fact $(u, v, w) \odot(u, v, w)^{*} \in X_{+}$ (if it holds), there exists an inverse element to $(u, v, w)$ with respect to the operation $\odot$.

Definition 3.4. The triple $(u, v, w) \in X^{3}$ is called to be invertible if $(u, v, w) \odot$ $(u, v, w)^{*}$ belongs to $X_{+}$. We denote the set of all invertible elements by $X_{i}^{3}$.

There is given an inverse element with respect to multiplication $\odot$ for an invertible element $(u, v, w) \in X_{i}^{3}$ as follows

$$
\begin{aligned}
(u, v, w)^{-1} & =\left((u, v, w) \odot(u, v, w)^{*}\right)^{-1}(u, v, w)^{*} \\
& \cong \frac{2}{(u-v)^{2}+(v-w)^{2}+(w-u)^{2}}(u, w, v),
\end{aligned}
$$

where the fraction is in the sense of double numbers multiplication.

Now we define the operation of division in the following way:

for

$$
(u, v, w) \oslash(x, y, z)=(u, v, w) \odot(x, y, z)^{-1}
$$

$$
(u, v, w) \in X^{3} \quad \text { and } \quad(x, y, z) \in X_{i}^{3} .
$$

\subsection{Equivalence classes with respect to the relation $\cong$}

Definition 3.5. The system

$$
\mathcal{M}:=\left(X^{3} \backslash \cong, \oplus, \odot\right)
$$

is called to be the three-polar space over the semi-field $X$.

In this part it will be proved that all operations in the introduced system $\mathcal{M}$ are well defined.

ThEOREM 3.6. Let $(u, v, w),(x, y, z),(a, b, c) \in X^{3}$ and $(u, v, w) \cong(a, b, c)$. Then the following:

(i) $(u, v, w) \cong(0,0,0)$ if and only if $(u, v, w)=(d, d, d)$ for some $d \in X$, 


\section{THREE-POLAR SPACE OVER THE SEMI-FIELD OF DOUBLE NUMBERS}

(ii) $(x, y, z) \oplus(u, v, w) \cong(x, y, z) \oplus(a, b, c)$,

(iii) $(x, y, z) \ominus(u, v, w) \cong(x, y, z) \ominus(a, b, c)$,

(iv) $(x, y, z) \odot(u, v, w) \cong(x, y, z) \odot(a, b, c)$,

(v) $(u, v, w)^{*} \cong(a, b, c)^{*}$,

(vi) $(u, v, w)$ is invertible if and only if $(a, b, c)$ is so,

(vii) if $(u, v, w)$ is invertible, then $(x, y, z) \oslash(u, v, w) \cong(x, y, z) \oslash(a, b, c)$. Proof.

(i) Directly from the definition of the relation $\cong$.

(ii), (iii), (v) are obvious.

(iv) Without loss of generality, let $(a, b, c)=(u+d, v+d, w+d)$ for some $d \in X$ in the rest of the proof. Then from distributivity of multiplication over addition

$$
\begin{aligned}
(x, y, z) \odot(a, b, c) & =(x, y, z) \odot(u+d, v+d, w+d) \\
& =[(x, y, z) \odot(u, v, w)] \oplus[(x, y, z) \odot(d, d, d)] \\
& \cong(x, y, z) \odot(u, v, w),
\end{aligned}
$$

since, by (i),

$$
\begin{aligned}
(x, y, z) \odot(d, d, d) & =(x d+y d+z d, x d+y d+z d, x d+y d+z d) \\
& \cong(0,0,0) .
\end{aligned}
$$

(vi) Let $(u, v, w)$ not be invertible. Then

$$
u_{1}+u_{2}=v_{1}+v_{2}=w_{1}+w_{2} \quad \text { or } \quad u_{1}-u_{2}=v_{1}-v_{2}=w_{1}-w_{2} .
$$

The proof follows by

$$
\begin{array}{lll}
a_{1}=u_{1}+d_{1}, & b_{1}=v_{1}+d_{1}, & c_{1}=w_{1}+d_{1}, \\
a_{2}=u_{2}+d_{2}, & b_{2}=v_{2}+d_{2}, & c_{2}=w_{2}+d_{2} .
\end{array}
$$

(vii) We have

$$
\begin{aligned}
& (a, b, c)^{-1} \\
& =\frac{2(u+d, w+d, v+d)}{[(u+d)-(v+d)]^{2}+[(v+d)-(w+d)]^{2}+[(w+d)-(u+d)]^{2}} \\
& =\frac{2(u, v, w)}{(u-v)^{2}+(v-w)^{2}+(w-u)^{2}} \oplus \frac{2(d, d, d)}{(u-v)^{2}+(v-w)^{2}+(w-u)^{2}} \\
& \cong \frac{2}{(u-v)^{2}+(v-w)^{2}+(w-u)^{2}}(u, v, w) \\
& =(u, v, w)^{-1} \quad \text { for } \quad(a, b, c)=(u+d, v+d, w+d), \quad d \in X .
\end{aligned}
$$




\section{TOMÁŠ GREGOR}

By the previous lemma, binary operations of addition, subtraction and multiplication are all independent of the choice of representatives, and thus they are all well defined. The properties of the system $\mathcal{M}$ are collected in the following statement.

LEMMA 3.7. The system $\mathcal{M}$ is a commutative ring with unit.

\section{Cancellation law in split-quaternions}

In this section, we suppose the operators $A, B, C$ in the form

$$
B_{i}: \mathbb{R}^{4} \rightarrow \mathbb{R}^{4}, \quad i=1,2,3,
$$

where $B_{1,2,3}=A, B, C$ are square matrices $4 \times 4$ with the real coefficients.

According to (11), we obtain the following equations:

$$
\begin{aligned}
& B_{1} B_{1}=B_{2} B_{3}=B_{3} B_{2}=B_{1}, \\
& B_{1} B_{2}=B_{2} B_{1}=B_{3} B_{3}=B_{2}, \\
& B_{1} B_{3}=B_{2} B_{2}=B_{3} B_{1}=B_{3},
\end{aligned}
$$

where multiplication of matrices is understood in the usual sense. We assume that $A=B_{1}$ is an identity matrix. This way, the system (9) is reduced to the following four equations:

$$
B_{2} B_{3}=B_{1}, \quad B_{3} B_{2}=B_{1}, \quad B_{3} B_{3}=B_{2}, \quad B_{2} B_{2}=B_{3} .
$$

These equations lead to a system of 48 non-linear equations with 32 unknown. We do not have a complete solution of this equation system. We considered only a special cases of the so-called split-quaternions.

The operation of multiplication of two split-quaternions $\left(x_{1}, x_{2}, x_{3}, x_{4}\right) \in \mathbb{R}^{4}$ and $\left(y_{1}, y_{2}, y_{3}, y_{4}\right) \in \mathbb{R}^{4}$ can be expressed in the "matrix-vector" notation as

$$
\left(x_{1}, x_{2}, x_{3}, x_{4}\right) \cdot\left(y_{1}, y_{2}, y_{3}, y_{4}\right)=\left(\begin{array}{cccc}
x_{1} & -x_{2} & x_{3} & x_{4} \\
x_{2} & x_{1} & -x_{4} & x_{3} \\
x_{3} & -x_{4} & x_{1} & x_{2} \\
x_{4} & x_{3} & -x_{2} & x_{1}
\end{array}\right)\left(\begin{array}{l}
y_{1} \\
y_{2} \\
y_{3} \\
y_{4}
\end{array}\right) .
$$

Then the operators $A_{2}, A_{3}$ can be represented by quadruples of real numbers $\left(b_{1}, b_{2}, b_{3}, b_{4}\right)$ and $\left(c_{1}, c_{2}, c_{3}, c_{4}\right)$, respectively. The operator $A_{1}$ is represented by $(1,0,0,0)$.

In this case, the system (10) has only 8 unknown and is easy solvable. It was solved by Mathematica. All solutions are of two kinds. The first one is not interesting trivial solution

$$
A_{2}=A_{3}=(1,0,0,0) .
$$




\section{THREE-POLAR SPACE OVER THE SEMI-FIELD OF DOUBLE NUMBERS}

The second type of solution is parametrizable

$$
\begin{aligned}
& A_{2}(r, s, t)=\left(-\frac{1}{2}, \frac{r}{2} \sqrt{3+4 s^{2}+4 t^{2}}, s, t\right), \\
& A_{3}(r, s, t)=\left(-\frac{1}{2},-\frac{r}{2} \sqrt{3+4 s^{2}+4 t^{2}},-s,-t\right),
\end{aligned}
$$

where $r \in\{1,-1\}, s \in \mathbb{R}, t \in \mathbb{R}$.

If $s=t=0$, then operators $A_{2}, A_{3}$ correspond to rotation in the complex plane by angle $2 \pi / 3$ and $4 \pi / 3$, respectively.

We observe that for all $x \in \mathbb{R}^{4}$,

$$
A_{1} \cdot x+A_{2}(r, s, t) \cdot x+A_{3}(r, s, t) \cdot x=(0,0,0,0)
$$

for all admissible $r, s, t$. Thus the equation (12) has a form of the cancellation law.

\section{Commutative rings on the set $\mathbb{R}^{4}$}

In the Section 3, we have constructed a class of commutative rings of three-polar spaces over the semi-field of double numbers. In the previous section, we have found the examples of polar operators that can be used to construct a three-polar space. In this section, we show how the operations from the threepolar space can be transfered to operations on the set $\mathbb{R}^{4}$.

Let $m: \mathcal{M}=X^{3} \rightarrow \mathbb{R}^{4}$,

$$
(a, b, c) \mapsto A_{1} \cdot\left(a_{1}, a_{2}, 0,0\right)+A_{2}(r, s, t) \cdot\left(b_{1}, b_{2}, 0,0\right)+A_{3}(r, s, t) \cdot\left(c_{1}, c_{2}, 0,0\right),
$$

where $a=\left(a_{1}, a_{2}\right) \in X, b=\left(b_{1}, b_{2}\right) \in X, c=\left(c_{1}, c_{2}\right) \in X$. The mapping $m$ is independent of the choice of representatives of equivalence classes $\mathbb{M}=\mathcal{M} \backslash \cong$. Thus it can be considered as a mapping $\mathbb{M} \rightarrow \mathbb{R}^{4}$ that is injective. If $s^{2}+t^{2}>0$, then the mapping $m$ is onto. Moreover, $m$ is linear, i.e.,

$$
\begin{aligned}
m((u, v, w) \oplus(x, y, z)) & =m(u, v, w)+m(x, y, z), \\
m(k \odot(u, v, w)) & =k_{1} m(u, v, w),
\end{aligned}
$$

for all $(u, v, w),(x, y, z) \in X^{3}, \quad k=\left(k_{1}, 0\right) \in X, \quad k_{1} \in \mathbb{R}$.

If $s^{2}+t^{2}>0$, then $m$ is a bijection, thus there is an inverse mapping, denoted by $n: \mathbb{R}^{4} \rightarrow \mathbb{M}$. Also this mapping is linear. Through these two mappings we can introduce new operations, say addition and multiplication, on $\mathbb{R}^{4}$ as follows:

$$
\begin{aligned}
& p \boxplus q=m(n(p) \oplus n(q)), \\
& p \boxminus q=m(n(p) \odot n(q)), \quad p, q \in \mathbb{R}^{4} .
\end{aligned}
$$

Immediately, by linearity of both mappings,

$$
p \boxplus q=p+q,
$$

where + is usual coordinate-wise addition in $\mathbb{R}^{4}$. 


\section{TOMÁŠ GREGOR}

More interesting situation arises from the operation of multiplication. Let us denote four base elements in $\mathbb{R}^{4}$ as $e_{1}, e_{2}, e_{3}, e_{4}$ and let

$$
p=\sum_{i=1}^{4} p_{i} e_{i}, \quad q=\sum_{j=1}^{4} q_{j} e_{j} .
$$

By properties of operations $\oplus, \odot$ and mappings $m, n$,

$$
\begin{aligned}
m(n(p) \odot n(q)) & =m\left(n\left(\sum_{i=1}^{4} p_{i} e_{i}\right) \odot n\left(\sum_{j=1}^{4} q_{j} e_{j}\right)\right) \\
& =m\left(\bigoplus_{i, j=1}^{4} p_{i} q_{j}\left[n\left(e_{i}\right) \odot n\left(e_{j}\right)\right]\right) \\
& =\sum_{i, j=1}^{4} p_{i} q_{j} m\left(n\left(e_{i}\right) \odot n\left(e_{j}\right)\right),
\end{aligned}
$$

where $\bigoplus$ means the sum with respect to $\oplus$. Therefore

$$
p \bullet q=\sum_{i, j=1}^{4} p_{i} q_{j}\left(e_{i} \bullet e_{j}\right),
$$

and thus it suffices to find the elements $e_{i} \bullet e_{j}=m\left(n\left(e_{i}\right) \odot n\left(e_{j}\right)\right)$.

Remark 5.1. It follows from the properties of the three-polar operations $\oplus$ and $\odot$, and the mappings $m$ and $n$ that the system $\left(\mathbb{R}^{4}, \boxplus, \bullet\right)$ is a commutative ring with unit $(1,0,0,0)$ regardless of the parameters $r, s, t$, except for the condition $s^{2}+t^{2}>0$. If $s=t=0$, then the mapping $m: X^{3} \rightarrow \mathbb{R}^{4}$ is not surjective and thus the mapping $n$ is not well defined.

EXAMPle 5.2. For $r=1, s=1, t=0$, c.f. (11), we have

$$
A=(1,0,0,0), \quad B=\left(-\frac{1}{2}, \frac{\sqrt{7}}{2}, 1,0\right), \quad C=\left(-\frac{1}{2},-\frac{\sqrt{7}}{2},-1,0\right)
$$

and the operation of multiplication in $\mathbb{R}^{4}$ possesses the following form

$$
\begin{aligned}
p \boxminus q=\quad & \left(p_{1} q_{1}+p_{2} q_{2}+\frac{9}{2} p_{3} q_{3}-\frac{5}{2} p_{4} q_{4}-\sqrt{7}\left(p_{3} q_{2}+p_{2} q_{3}\right),\right. \\
& p_{1} q_{2}+p_{2} q_{1}-\sqrt{7}\left(p_{2} q_{4}+p_{4} q_{2}\right)+\frac{5}{2}\left(p_{3} q_{4}+p_{4} q_{3}\right), \\
& p_{1} q_{3}+p_{3} q_{1}-p_{2} q_{4}-p_{4} q_{2}, \\
& \left.p_{1} q_{4}+p_{4} q_{1}-p_{2} q_{3}-p_{3} q_{2}+\sqrt{7}\left(p_{3} q_{3}-p_{4} q_{4}\right)\right) .
\end{aligned}
$$




\section{THREE-POLAR SPACE OVER THE SEMI-FIELD OF DOUBLE NUMBERS}

\section{Conclusions}

Multi-polarity has applications in physics. There are known multi-phase electric current, multi-polar magnets, oscillators, etc. Colours are another possible field of application of multi-polarity. The mathematical structure of multi-polar spaces is described in the paper [4. Here is presented a multi-polar space over the semi-field of all non-negative real numbers. In this paper, we investigated a multipolar space over a more general semi-field - the semi-field of double numbers. We studied a special case when polar operators are presented as split-quaternion multiplication. In this case we proved that the multi-polar space possesses structure of commutative ring with unit. Moreover, this system can be isomorphically transferred to $\mathbb{R}^{4}$. The operation of addition is usual coordinate-wise addition. Converse problem, to obtain an arbitrary commutative and associative operation on $\mathbb{R}^{4}$ distributing over coordinate-wise addition from a multi-polar space, remains open.

\section{REFERENCES}

[1] ANTONUCCIO, F.: Semicomplex analysis and mathematical physics (corrected version), ArXiv General Relativity and Quantum Cosmology e-prints, 1993, http://arxiv.org/abs/gr-qc/9311032

[2] DEMIR, S.-TANIŞLI, M.-KANSU, M. E.: Generalized hyperbolic octonion formulation for the fields of massive dyons and gravito-dyons, Internat. J. Theoret. Phys. 52 (2013), 3696-3711, http://dx.doi.org/10.1007/s10773-013-1675-3.

[3] DEMIR, S.-TANIŞLI, M.-TOLAN, T.: Octonic gravitational field equations, Internat. J. Modern Phys. A 28 (2013), http://www. worldscientific.com/doi/abs/10.1142/S0217751X13501121

[4] GREGOR, T.-HALUSKA, J.: Lexicographical ordering and field operations in the complex plane, Matematychni Studii 41 (2014), 123-133, http://matstud.org.ua/texts/2014/41_2/123-133.html

[5] KISIL, V. V.: Erlangen Programme at Large: An Overview, ArXiv e-prints, archivePrefix 2011, http://arxiv.org/abs/1106.1686

[6] LENSKI, V.: Generation of multipolar electromagnetic energy, 2008, http://www.lens.org/lens/patent/US_2008_0112111_A1.

[7] TANIŞLI, M.-KANSU, M. E.-DEMIR, S.: Reformulation of electromagnetic and gravito-electromagnetic equations for Lorentz system with octonion algebra, Gen. Relativity Gravitation 46 (2014), http://dx.doi.org/10.1007/s10714-014-1739-6.

Mathematical Institute Slovak Academy of Sciences extension in Košice

Grešákova 6

04001 Košice

SLOVAKIA 